\title{
Two Dogmas of the Artistic-Ethical Interaction Debate - Erratum
}

\author{
Louise Hanson
}

DOI: 10.1017/can.2019.13, Published by Cambridge University Press, 24 October 2019.

In the original publication of this article (Hanson 2019) two of the references by Harold were erroneously misattributed, and the name of reference Giovannelli was misspelled. The article has since been corrected.

\section{Reference}

Hanson, Louise. 2019. “Two Dogmas of the Artistic-Ethical Interaction Debate." Canadian Journal of Philosophy. DOI: $10.1017 /$ can.2019.13 